\title{
Analysis of the Waveform of the Acoustic Emission Signal via Analogue Modulations with Neural Networks
}

\author{
Orlando Gea ${ }^{1}$, Luiz P. Calôba ${ }^{2}$ \\ ${ }^{1,2}$ (Electrical Engineering Department, Federal University of Rio de Janeiro, Brazil)
}

\begin{abstract}
The acoustic emission (AE) technique is a non-destructive testing technique applied to pressurized rigid pipelines in order to identify metallurgical discontinuities. This study analyses the dynamic behavior of the propagation of discontinuities in cracks via AE, in the following propagation classes: no propagation (NP), stable propagation (SP), and unstable propagation (UP). The methodology involves applying the concept of modulation of analogue signals, which are used in signal transmission in telecommunications, for the development of a neural network in order to determine new parameters for the AE waveform. The classification of AE signals into propagation classes occurs, therefore, through the extraction of information related to the dynamics of AE signals, by means of the parameters of the analogue carriers of the modulations (in amplitude and in angle) that make up the AE signal. This set of parameters enables an efficient classification (average of 90\%) through the identification of patterns from the AE signals in the classes for monitoring the state of the discontinuity, through the use of computational intelligence techniques (artificial neural networks and nonlinear classification).
\end{abstract}

Keywords: acoustic emission, analogue modulation, neural network, non-destructive testing.

\section{Introduction}

Detection of the propagation of metallurgical defects in pressurized pipelines by means of the nondestructive acoustic emission (AE) testing technique is important in preventive inspection. However, the operational difficulty is in the classification of the AE signals into the different states of the crack (propagation classes). The study of artificial neural networks is done in order to improve identification of the behaviour of crack discontinuity in terms of its influence on the integrity of the structure subjected to the internal pressure [15]. The discontinuity, when subjected to a load, may become a defect-carrying area, and thus an area of stress concentration which, due to stimulation, leads to a redistribution of localized stresses, with the quick release of mechanical stress waves, which are located internally in the material in the form of transient mechanical waves. Thus, the biggest contribution of the technique is the analysis of the dynamic behaviour of the discontinuities, which enables the inspection of in-service equipment in order to evaluate the presence of discontinuities that could compromise the equipment's integrity.

Neural networks can be applied to systems modelling and pattern recognition, due to the process of learning by parallel and distributed processing. Thus, the learning of complex input-output mappings is made possible, due to the ability to learn from data with or without a teacher (supervised and unsupervised training). Knowledge is acquired from its environment via the learning process and it is stored in the neural connections (synapses or synaptic weights).The main motivation of this work is the modelling of the AE phenomenon as a response of a dynamic system consisting of modulated signals (analogue modulations) determined by a neural network to provide, as a result, the classification into one of the three possible propagation classes for the crack: no propagation (NP), stable propagation (SP), and unstable propagation (UP). In telecommunications, the modulation is used from communication systems by carriers to shift the frequency spectrum of a signal, because modulation consists of the process by which certain characteristics of the carrier are modified by a waveform, known as a modulator, $m(\mathrm{t})$. In the case of the carrier being sinusoidal $-\beta(\mathrm{t}) \cos [\alpha(\mathrm{t})]-$ the modulation is the continuous wave $(\mathrm{CW})$ type - see Figure 1.

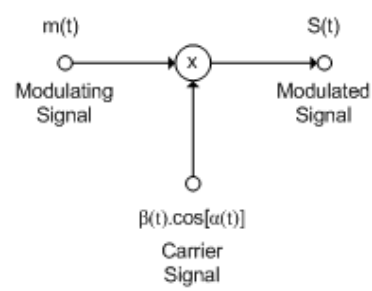

Figure 1 - Modulation process 
Analogue modulation can be classified by amplitude (linear), if the parameter in question is the amplitude of the signal, $\beta(\mathrm{t})$; or by angle (non-linear), if the parameters in question are the frequency (FM) and/or the phase (PM) of the signal, $\alpha(\mathrm{t})$. Thus, the modulations cause the linear variation of the synodic parameters (amplitude, frequency, and phase), in accordance with the modulating signal, and in turn as per the modulation type (Figure 2).
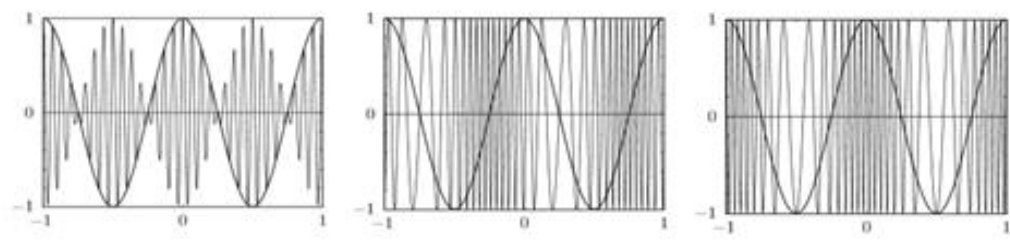

Figure 2 - (a) linear modulation (AM) and (b) non-linear modulation (PM and FM) for the signal $m(\mathrm{t})=$ $\cos (2 \pi \mathrm{t})$

Visualization of the AE signal as the envelope of a composite signal - superposition of modulated signals by carriers of the analogue modulation processes of a given modulating signal — introduces a set of new features for analysis of the parameters which constitute the carriers and the modulating signal. These parameters represent the transition between the $\mathrm{AE}$ signals during the hydrostatic testing and used as inputs for neural networks, with the objective of identifying patterns among the propagation classes of discontinuities (NP, SP, and UP). The provision of the information regarding the propagation classes becomes an important aid in the non-destructive mechanical tests for the inspection of rigid pipes via $\mathrm{AE}$.

\section{Modelling}

Modulation changes the format of a signal, based on another, by the variation of defining characteristics. In an amplitude modulation (AM), the signal to be transmitted (modulating), $m(t)$, influences the amplitude of a frequency carrier, fam, so that the carrier's amplitude varies directly proportional to the amplitude of the signal. In phase modulation (PM) or frequency modulation (FM), the variation of the phase or frequency of the frequency carriers $-\mathrm{f}_{\mathrm{pm}}$ and $\mathrm{f}_{\mathrm{fm}}$, respectively - is directly proportional to the amplitude of the modulating signal. The following equations illustrate the concept for the modulations, in which $\operatorname{Sm}(\mathrm{t}), \mathrm{K}_{\mathrm{am}}, \mathrm{K}_{\mathrm{pm}}$, and $\mathrm{K}_{\mathrm{fm}}$ represent the modulated signal and the modulation constants for AM, PM, and FM, respectively [6,7].

Amplitude Modulation (AM)

$S_{m}(t)=\left(K_{a m}+m(t)\right) \cdot \cos \left(2 \pi \cdot f_{a m} \cdot t\right)$

Phase Modulation (PM)

$S_{m}(t)=A_{p m} \cdot \cos \left(2 \pi \cdot f_{p m} \cdot t+K_{p m} \cdot m(t)\right)$

Frequency Modulation (FM)

$S_{m}(t)=A_{f m} \cos \left[2 \pi\left(f_{f m} \cdot t+K_{f m} \int_{-\infty}^{t} m(\alpha) d \alpha\right]\right.$

Given the above information, Figure 3 illustrates the principle of the model.

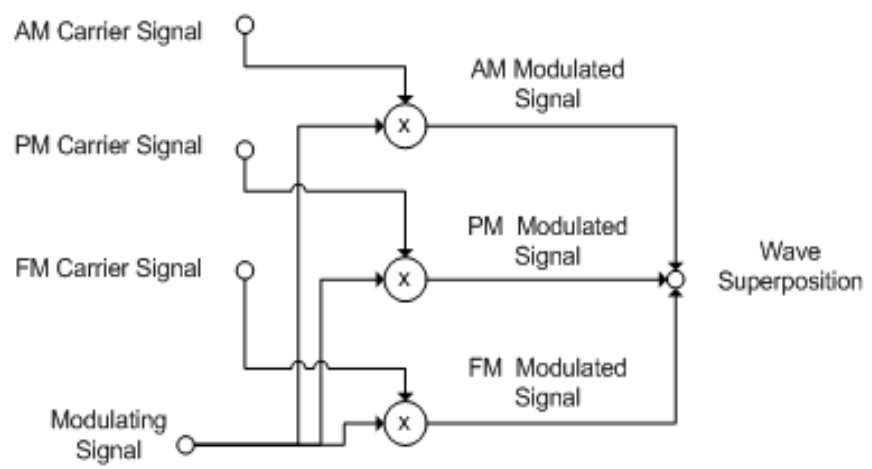

Figure 3 - Principal of the model 
Thus, the resulting signal, $\mathrm{F}(\mathrm{t})$, can be defined as a composition of $S_{m(i)}(\mathrm{t})$, with $\mathrm{a}_{0}, \mathrm{a}_{1}, \mathrm{a}_{2}$, and $\mathrm{a}_{3}$ constant, and $\mathrm{a}_{0}$ being the representation of the average of the signal, as can be seen in Equation 4:

$F(t)=a_{0}+a_{1} S_{m(a m)}(t)+a_{2} S_{m(p m)}(t)++a_{3} S_{m(f m)}(t)$

Due to the AE signal being a physical signal (causal signal), it only occurs for $t \geq 0(u(t)$ is a step function), as can be seen in Equation 5:

$E A(t) \cong F(t) \cdot u(t) \cong$

$\cong a_{0}+a_{1}\left[K_{a m}+m(t)\right] \cos \left(2 \pi f_{a m} t\right)+a_{2} A_{p m} \cos \left[2 \pi f_{p m} t+K_{p m} m(t)\right]+$

$+a_{3} A_{f m} \cos \left[2 \pi\left(f_{f m} t+K_{f m} \int_{0}^{t} m(\alpha) d \alpha\right)\right]$

Therefore, the function representing the AE signal will be a parametric function, as shown in Equation 6:

$E A(t) \cong a_{0}+a_{1} F\left[K_{a m}, f_{a m}, m(t)\right]++a_{2} F\left[K_{p m}, f_{p m}, m(t)\right]+a_{3} F\left[K_{f m}, f_{f m}, m(t)\right]$

The neural network was developed with the objective of estimating the constants of the parametric function (constants $K$ and $f$ of Equation 6), since the independent variable is time (t). This estimate of the neural network results in the identification of the signal's behaviour in relation to its dynamics, through the amplitude, through the frequency, and through the phase in the transition between the AE signals recorded. This presents a new visual perspective of the phenomenon.

Figure 4 presents the functional description of the elements which constitute the signal flow graph model of Equation 5 - the basic internal structure of the neural network developed. Figure 5 presents, based on signal flow (Figure 4), the structure of the modulations in functional blocks in the hybrid neural network.

The term $\mathrm{a}_{0}$ of the equations $(4,5$, and 6$)$ is disregarded in the structuring of the neural network, because in the studies of the AE signals captured, the average (expected value) approaches zero.
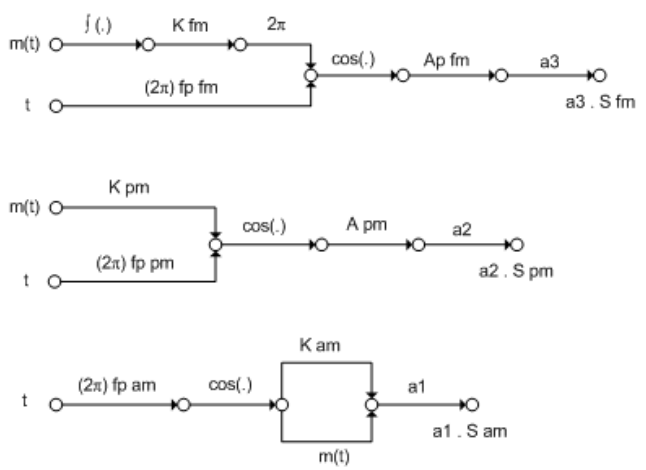

Figure 4 - Graph of signal flow

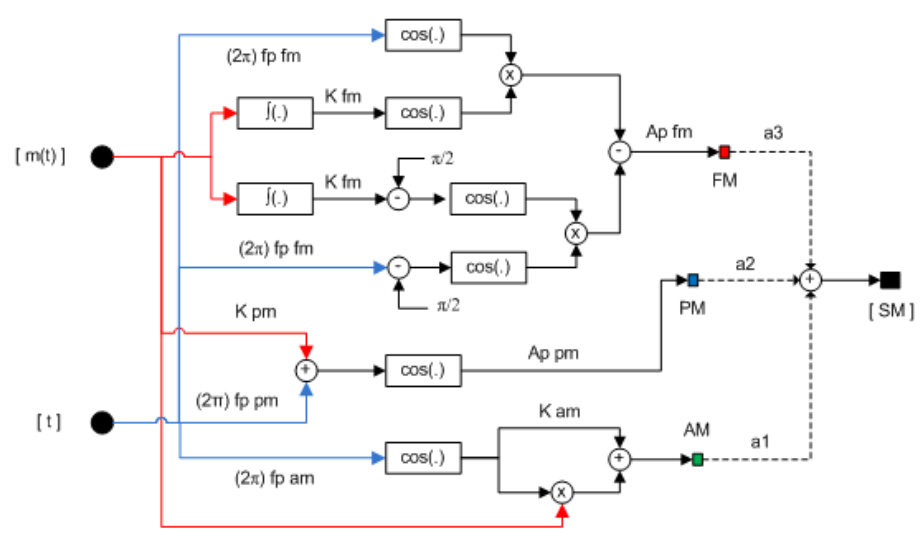

Figure 5 - Structure of the hybrid neural network with the pathways of the modulations for $\mathrm{F}(\mathrm{t})$ 
In the neural network, multiple neurons are interconnected to form a distributed network. The configuration of these interconnections in the hybrid neural network was structured based on the graph and directed towards the definition of the topology. In the hybrid neural network, each neural block becomes responsible for a load of the processing (internal parts of the hybrid network structure). This allows the output of each block to exhibit the function that the functional block represents. This strategy is based on the fact that a neural network does not necessarily achieve data symmetry, and the topological specification aspect of the network has, as a basis, the universal approximation theorem (Kolmogorov theorem), which provides the necessary premise for defining the structural settings of the neural blocks as "functional" in the hybrid network (approximation of analytical functions).

The hybrid neural network can be considered to be a four-layered network with characteristics of a network with time-delayed inputs. The algorithm was structured in accordance with the functioning principle of the Feedforward Neural Networks (Multilayer Perceptron Networks), in which each neuron is composed of two parts: activation potential function and activation function. The activation potential function determines how the inputs of the network are combined within the neuron in accordance with the synaptic weights, while the activation function provides the output of the neuron [8-11].

The implementation of the backpropagation training algorithm (descending gradient) is done across the hybrid network by pathways that represent each modulation (Figure 5). After the training, the synapses of the hybrid network supply the parameters of the carriers that make up the signal, by means of the connection mesh (synapses). It can be seen that the hybrid neural network is asymmetric compared to the typical structures of neural networks; therefore, the training is done internally in each neural network that constitutes a functional block (as in the whole hybrid network) through the local gradient function applied to each internal and external layer of the neural block and the hybrid network, respectively.

The characterization of the carriers by the neural network highlights behaviours that can help to understand the phenomena, because analysis by means of these carriers distributes information on aspects relevant to the modulations, in accordance with their relevance. In this type of neural network, the desired information is not in the output of the network, but in its structure in the form of synaptic weights that represent the parameters of the carriers. The hybrid neural network offers the best combination of AM, PM, and FM in the learning, through the reduction of the mean squared error (supervised training).

\section{Experimental Test}

The hydrostatic tests were performed on API-XL 60 specification steel specimens (pipeline segments) with seams, a nominal diameter of 20 inches $(50.8 \mathrm{~cm})$, length of $2.0 \mathrm{~m}$, and $14.5 \mathrm{~mm}$ thick walls. Their ends were machined to enable the assembly of two flanges for containment of the pressurization fluid (sealing with rubber o-rings). An external crack was machined at the centre of the pipe, and fatigue was subsequently introduced, via load cycles, in order to cause a semi-elliptical defect $160 \mathrm{~mm}$ long, $7.33 \mathrm{~mm}$ deep (ratio of around 20:1), and $1.1 \mathrm{~mm}$ wide. The specimen was considered to be a thin-walled reservoir, because the ratio between the thickness of the wall and the radius of the pipe was less than 0.1 . The specimen was submitted to a load by means of variation of the internal pressure, in accordance with the loading curve, up to the point equivalent to $90 \%$ of the yield strength of the material.

The behaviour of the crack was accompanied through the AE systems (VALLEN and PAC), the ultrasound was accompanied via time of flight diffraction (ToFD), while the extensometry was monitored via electrical resistance for recordings of the dimensions of the discontinuity in the crack during the hydrostatic testing. The AE system consisted of VALLEN and PAC sensors positioned as shown in Figure 6. A detection threshold of $45 \mathrm{~dB}$ was fixed in the PAC with resonant sensors at $150 \mathrm{kHz}$. For the VALLEN, a detection threshold of $65 \mathrm{~dB}$ was fixed for the resonant sensors at $150 \mathrm{kHz}$, with a sampling rate of $1 \mathrm{MHz}$ and 200 pretrigger samples. The ultrasound via ToFD was done with wedges of $10 \mathrm{MHz}$, transducers of $60^{\circ}$, and an acquisition rate of $1 \mathrm{~Hz}$. The VALLEN sensors distributed in the specimen recorded the AE signal waveforms during the test in accordance with the time distribution by sensor (Figure 6). The test behaviour (testing time, internal pressure, AE signals recorded by the VALLEN equipment, and the depth of the crack) is demonstrated by the graphs in Figures 7 and 8 . 


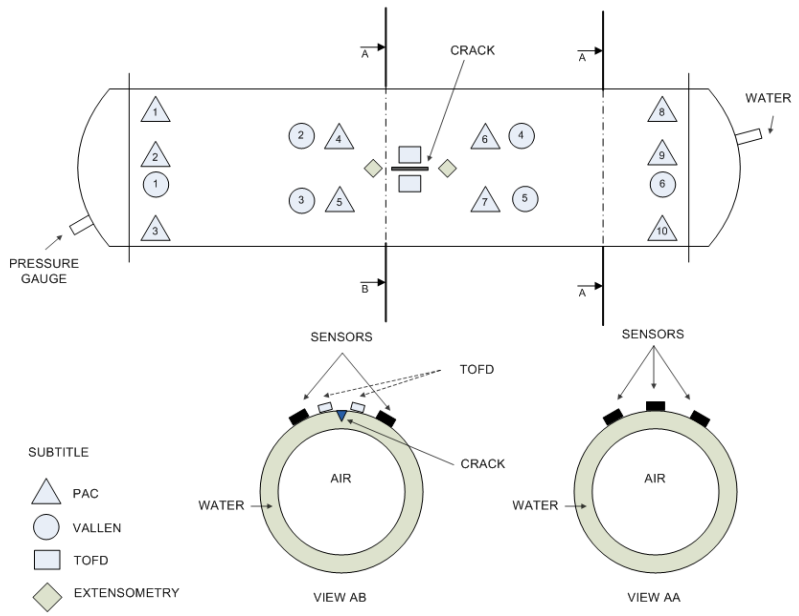

Figure 6. Structure of the specimen

Figure 7 shows the pressure curve, the depth of the crack during the hydrostatic testing, and the recordings of AE signals captured. Pressure levels were programmed during the pressurization cycle in the test. At these levels, the pressure was kept constant for a minimum of $10 \mathrm{~min}$; however, the last level was maintained for a minimum of $60 \mathrm{~min}$. The average incremental pressure rates during loading were $10.0 \mathrm{bar} / \mathrm{min}$ until the first level, and $2.5 \mathrm{bar} / \mathrm{min}$ at the other levels until reaching the nominal pressure equal to $90 \%$ of the yield strength of the pipe material planned for the last level.

The total rupture of the pipe occurred at the end of the last level of the first loading cycle, so it was not possible to perform the second loading cycle.

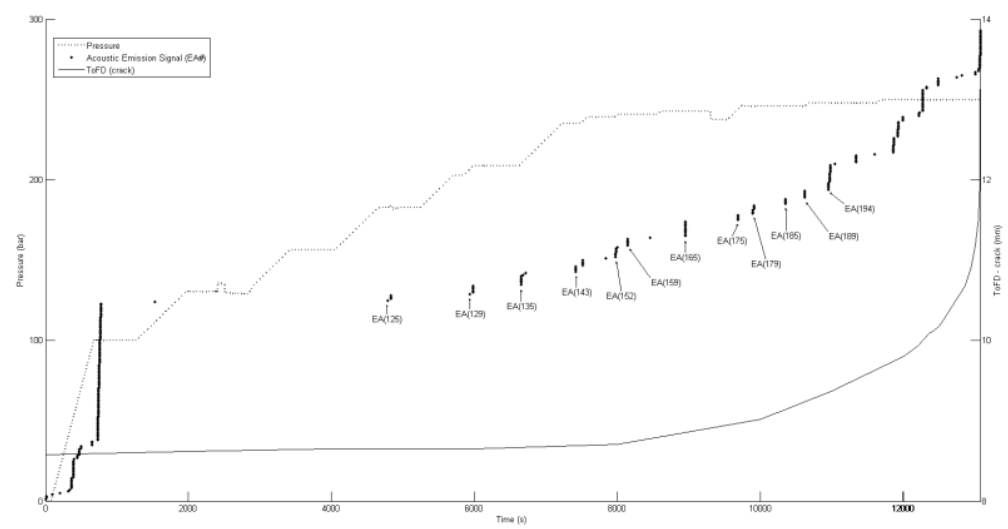

Figure 7 - Pressure distribution, ToFD, and AE recorded during the hydrostatic test $\left(1 \mathrm{bar}=0.980 \mathrm{kgf} / \mathrm{cm}^{2}\right)$.
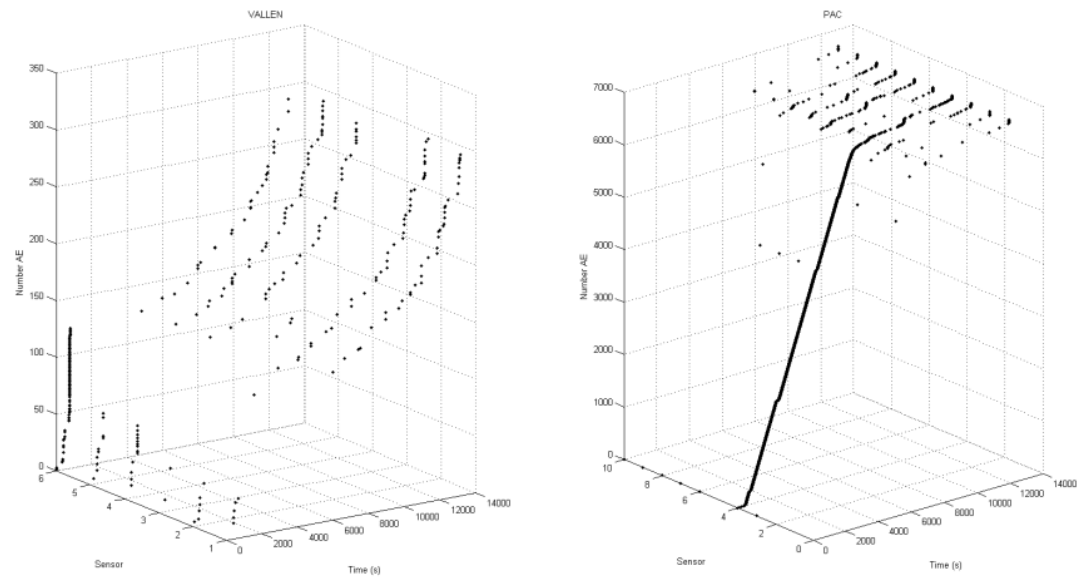

Figure 8 - AE recordings by sensor during the hydrostatic testing with the VALLEN and PAC equipment. 
The high number of signals (spurious signals) recorded at the beginning of the test (up until $2.10^{3} \mathrm{~s}$ ) $25 \%$ coming from sensors near the crack and $75 \%$ from sensors at the extremities of the specimen — was due to the adjustment of the sealing system and the entry of the water at the beginning of the pressurization.

The propagation classes of the discontinuity were defined based on the behaviour in the variation of the crack in accordance with the internal pressure variation (Table 1):

- No propagation (NP): The discontinuity of the crack undergoes minor changes in size for large pressure variations (metallurgical adjustment).

- Stable propagation (SP): The discontinuity of the crack undergoes changes in size for pressure variations (elastic effect).

- Unstable propagation (UP): The discontinuity of the crack undergoes significant changes in size for little or no pressure variation (inelastic or plastic effect).

Table 1 shows the quantitative distribution of the propagation classes for the discontinuity of the crack in the hydrostatic test.

Table 1.Statistics for the NP, SP, and UP classes.

\begin{tabular}{|l|l|l|l|}
\hline \multirow{2}{*}{} & \multicolumn{3}{|l|}{ Time interval $\left(.10^{3} \mathrm{~s}\right)$} \\
\cline { 2 - 4 } & {$[02,06]$} & {$[06,12]$} & {$[12,14]$} \\
\hline Variation of the crack & $0.34 \%$ & $13.29 \%$ & $35.40 \%$ \\
\hline Pressure variation & $59.87 \%$ & $19.63 \%$ & $0 \%$ \\
\hline Number of signals (centre of the pipe) & 7 & 65 & 40 \\
\hline Number of signals (extremities) & 3 & 40 & 22 \\
\hline
\end{tabular}

The need to study the EA phenomenon via new parameters based on the waveform is important because of the difficulty encountered in identifying patterns, even using the same recorded parameters (amplitude, rise time, energy, and count/duration) for equipment from different manufacturers (VALLEN and CAP), as illustrated in Figures 9 and 10. Currently, the parameters of this equipment are used in the analysis of $\mathrm{AE}$ signals in the search for cracks and/or faults in the integrity of rigid pipelines; however, without the characterization of the propagation classes.
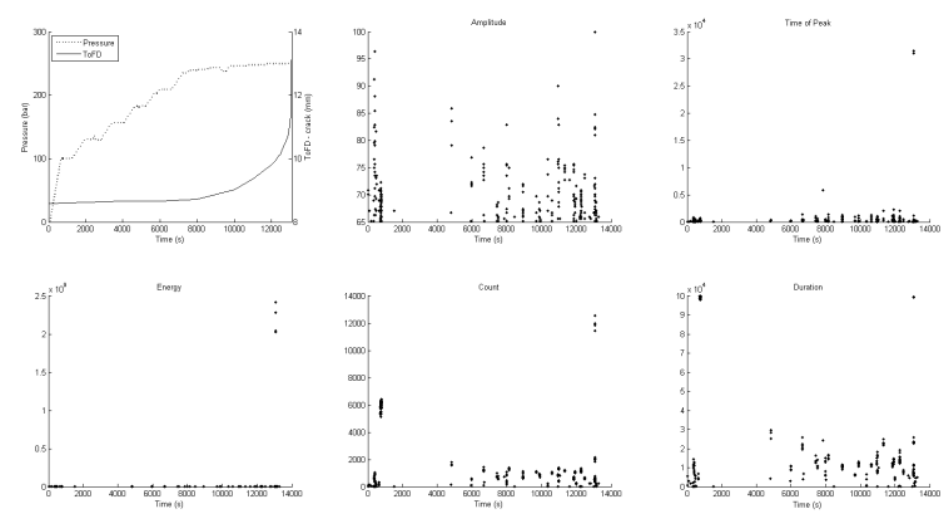

Figure 9 - VALLEN parameters
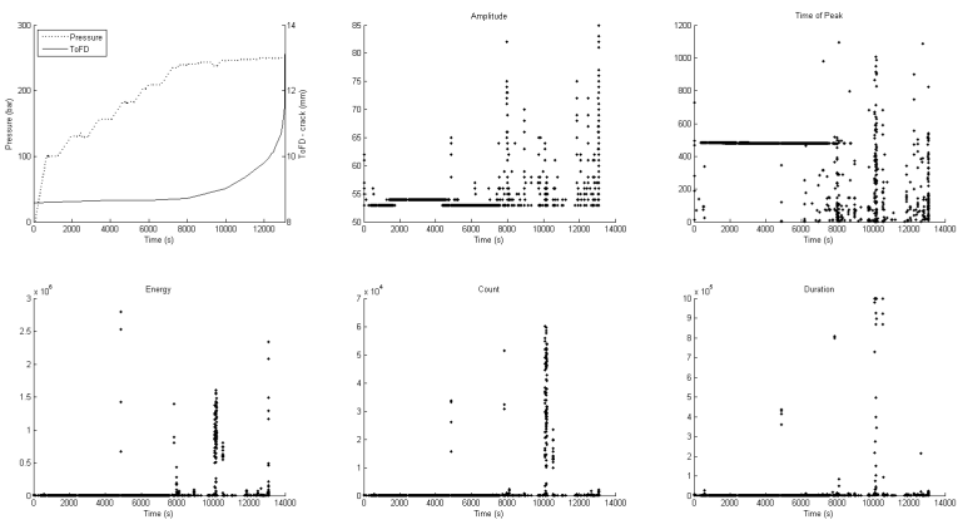

Figure 10 - PAC parameters 
Works using these parameters have been done using neural networks for the identification of the propagation classes [2-4].The difference between the distributions for the same parameters, measured by different equipment, results in inaccurate conclusions regarding the possible propagation regions of the discontinuity. Thus, analysis of the carrier parameters that make up the AE signal is important for the categorization of the states of the discontinuity in the crack, because they illustrate the transition phenomenon in the AE signals.

\section{Application Of The Methodology In The Testing}

Usage based on the signal decomposition technique aims for the identification of different classes in accordance with the behavior of the crack's propagation: no propagation (NP), stable propagation (SP), and unstable propagation (UP). The AE signals from the testing consist of a set of observations, $\left\{\mathrm{Z}\left[\mathrm{Y}_{\mathrm{i}}\right], \mathrm{i} \in \mathrm{N}\right\}$, in which the signal is a set $-\{\mathrm{Y}(\mathrm{t}), \mathrm{t} \in \mathrm{T}\}$ - and $\mathrm{T}=\left\{\mathrm{t}: \mathrm{t}_{1}<\mathrm{t}<\mathrm{t}_{2}\right\}$, as can be seen in Figures 11 and 12. The objective becomes, therefore, to understand the differences between the signals, and this enables the classes and their elements to be presented.

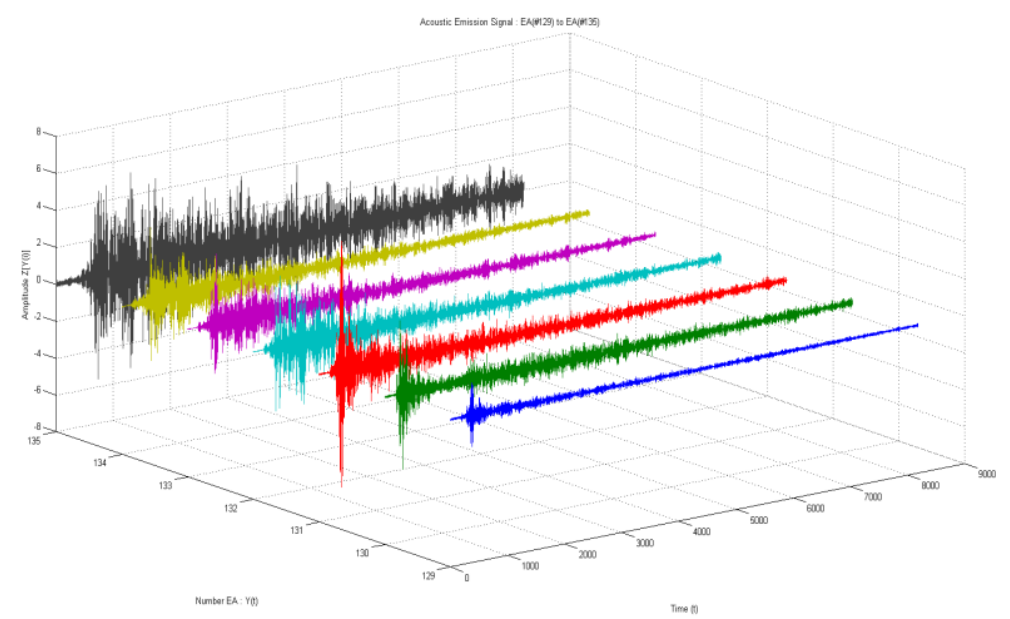

Figure 11 - Examples of AE signals in the SP class

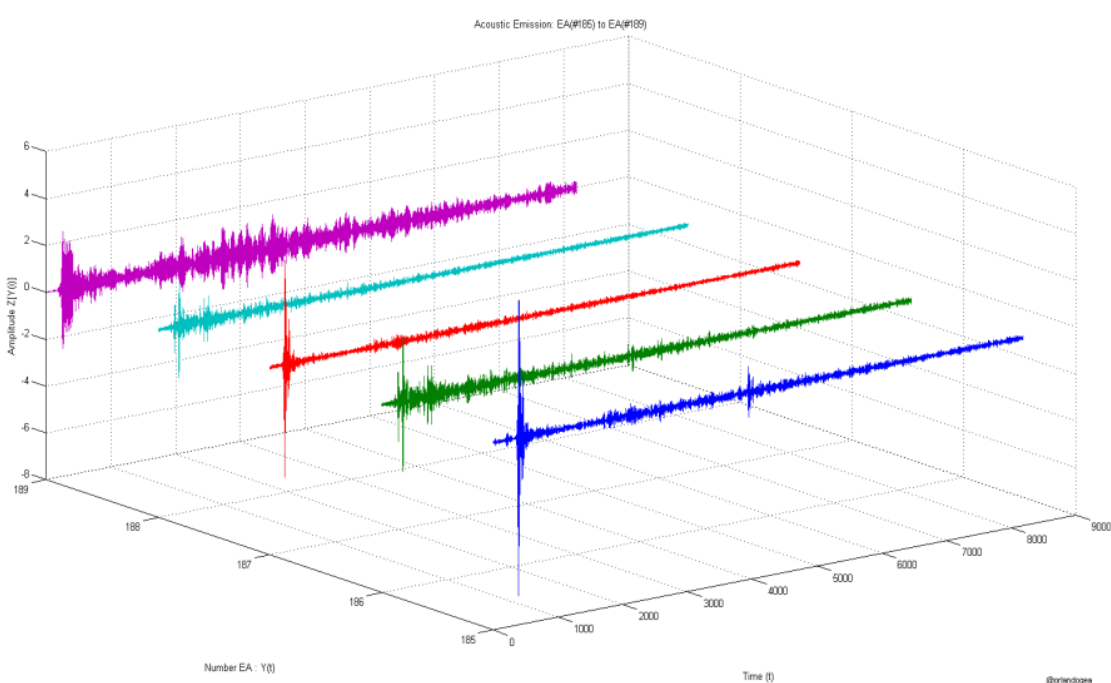

Figure 12 - Example of the AE signals in the UP class

The AE signals were analyzed in the frequency domain in groups (elements of the SP and UP classes), as per Figures 13 and 14, with a view to determining the size of the cut-off window in the original AE signal. The cut-off was done in order to improve the result of the hybrid neural network training, because more than $50 \%$ of the signal could be disregarded, with the boundary of the main spectrum predominating and the suppressed part assumed to be noise (Figure 15). 


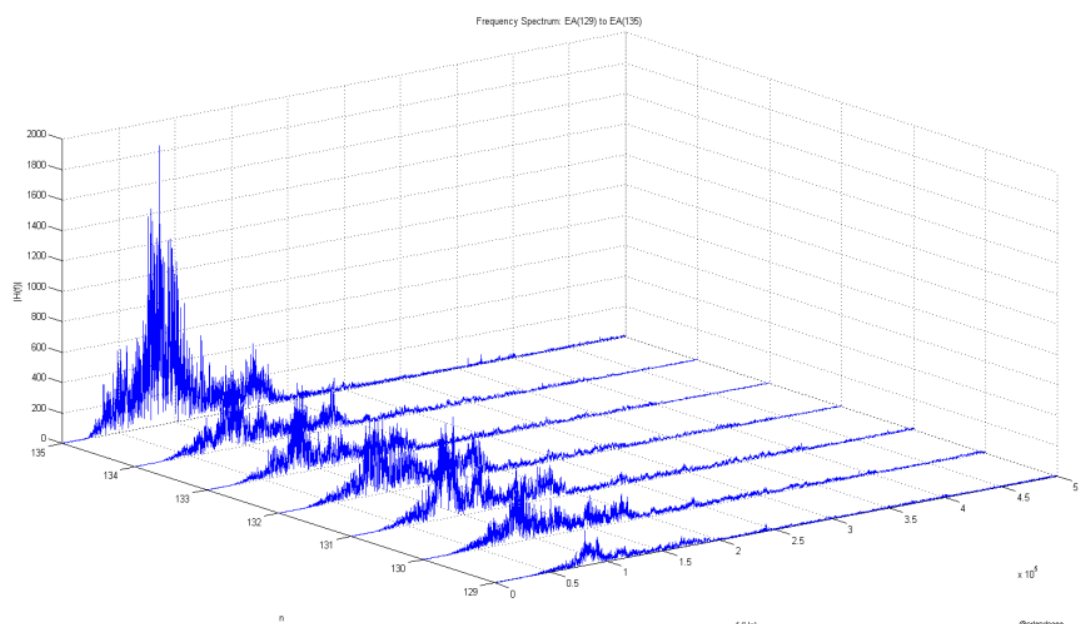

Figure 13 - Frequency spectrum of the AE group in SP (Figure 11)

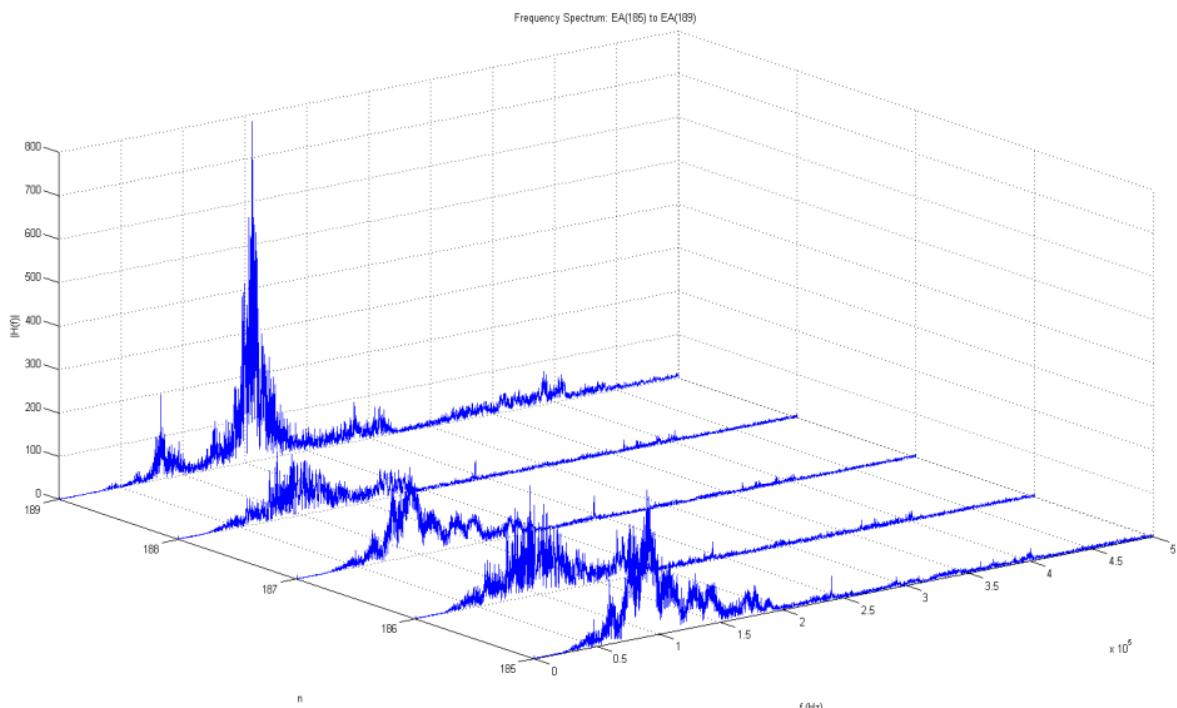

Figure 14 - Frequency spectrum of the AE group in UP (Figure 12)
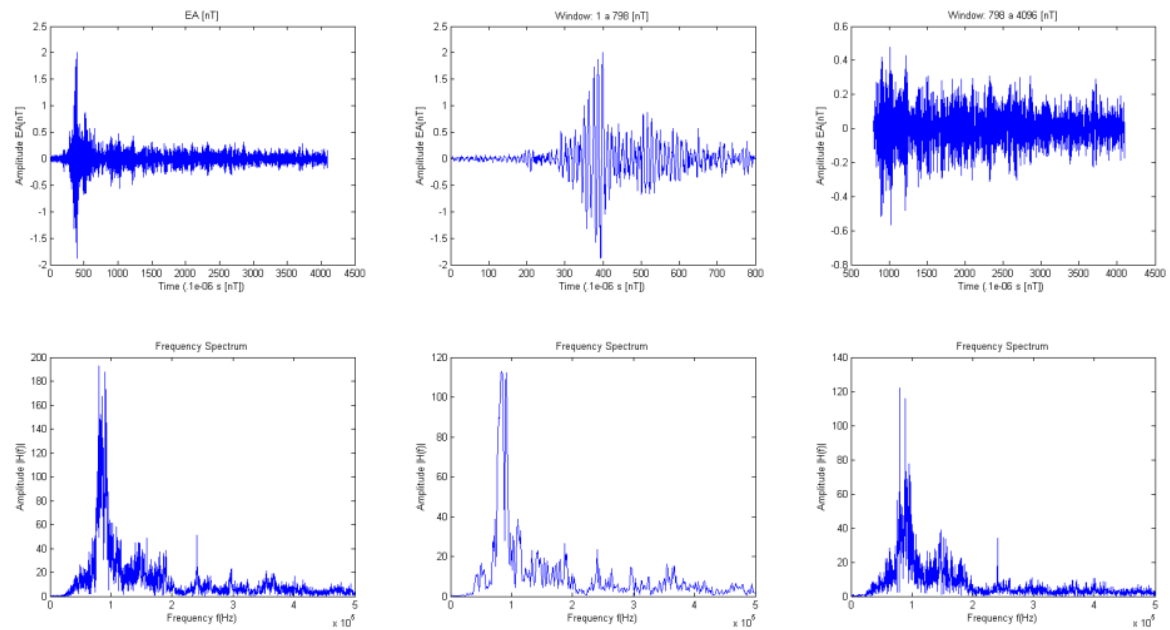

Figure 15 - Example of AE signal, cut-off window of the AE signal, and suppressed part (noise), as well as their respective frequency spectra. 
The hybrid network was trained (supervised training) with the AE signals in order to provide the set of carrier parameters that make up each recorded signal. These parameters consisted of a new data set, which presented the dynamics of the signals in the SP and UP regions. From the set of carrier parameters for each AE signal recorded during the hydrostatic testing, unsupervised learning techniques were applied to complete the identification of patterns among the propagation classes for the crack discontinuity:

- K-means method: the K-means algorithm is one method used to find groups and the centres of these groups, based on a data set with no prior classification. The idea is to partition a data set into separate groups, in which the Euclidean distance between the attributes vector and the representatives of the classes is used as a dissimilarity measure (Table 2 ).

- Self-organizing map: method that is particularly useful when there is non-linear mapping inherent in the problem itself. Enables the transformation of an arbitrarily-sized input standard into a two-dimensional discrete map, in a topologically adaptive manner (Figure 16).

Table 2. Average percentage of hits with k-means, for classification of the AE signals based on the carrier

parameters.
\begin{tabular}{|l|l|}
\hline SP & UP \\
\hline $91.2 \%$ & $94.3 \%$ \\
\hline
\end{tabular}

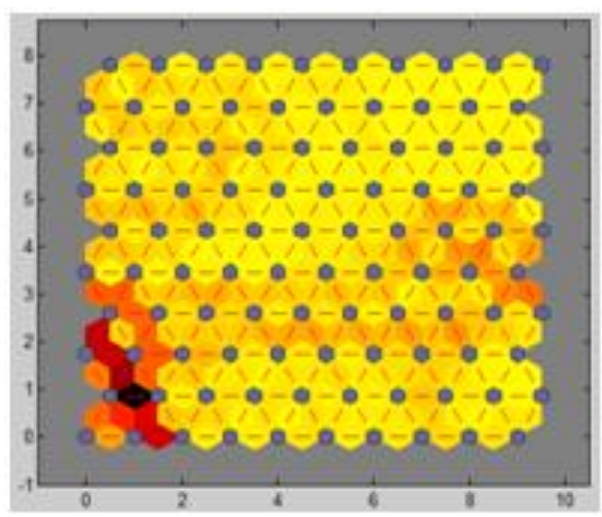

Figure 16 - Example of map resulting from the parameters of the signals in the SP class.

The non-supervised techniques were used based on the parameter set established by the hybrid neural network during the supervised training, for the signals of both the SP and UP classes (Table 1). The error percentage via the K-means method is basically concentrated in the transition region between the SP and UP classes (Table 1 and Figure 7). This transition region is also illustrated in the self-organizing map - see Figure 16. The transition region (Table 1) for this study was defined on the basis of the experience of experts in the area of instrumentation. Thus, any changes in this area will bring new results, and, therefore, part of the studies involves the best definition of the transition range [1-4]. For the purposes of metallurgical inspection using the $\mathrm{AE}$ method, the main objective of using the technique with the neural network is the knowledge of the transition phase between the SP and UP classes, based on new characteristics, because this reduces the likelihood of damage to the integrity of the pressurized pipe occurring during operation (UP class).

\section{Conclusion}

The results were satisfactory, including for both the controlled test of the model (with deterministic signals applied to the network) and for the AE signals (stochastic signals) presented to the hybrid network. The hybrid network represented a neural circuit structured in functional blocks (neural networks), with direct and indirect influence between them due to the segregation of activities by neural block. The network exhibited results for the context signals, both by the transition analysis (compared with the previous signal) and by the analysis based on a deterministic signal (compared with a given signal). The decomposition of the signal into three other waveforms, modelled by the carriers of analogue modulations, enables the characteristics of the phenomenon to be visualized. Modulation and demodulation are usually done via complex circuit techniques; however, the hybrid neural network presented a practical and efficient solution for the application of this technique utilized in telecommunications and signal processing. The AM modulation exhibits the characteristics of the phenomenon's amplitude in a new waveform, while the same principle is identified by the PM and FM modulations in relation to the phase and frequency requirements, respectively. Through the incorporation of time into the operation of a neural network, the network becomes capable of perceiving the statistical variations in non-stationary processes. 
The implicit representation of time enables a static neural network (multilayer perceptron) with dynamic properties (with short-term memory). Thus, it is an approach for a nonlinear dynamic system, in which the static network is responsible for the nonlinearity and the memory is responsible for the time.

\section{Acknowledgements}

The Carlos Chagas Filho Research Support Foundation of the State of Rio de Janeiro (FAPERJ) and the Coordination for the Improvement of Higher Education Personnel (CAPES), for the financial support via scholarships; the Leopoldo Américo Miguez de Mello Centre for Research and Development (CENPES) of PETROBRAS for enabling the research and development project in partnership with COPPE-UFRJ and UFRGS; and the LAMEF at UFRGS for the technical and operational support in the hydrostatic tests.

\section{References}

[1]. C F C Pinto, R R da Silva, L P Calôba and S D Soares, Probability of defect propagation in pipelines (POP curves), Insight, 56 (4), pp 1-7, 2014.

[2]. R R Silva, L P Calôba, M H S Siqueira, L V S Sagrilo and J M A Rebello, Detection of the Propagation of Defects in Pressurized Pipes through the Acoustic Emission Technique Using Artificial Neural Networks, ECNDT - Poster 1, pp 1-17, 2006

[3]. R R Silva, S D Soares, L P Calôba, M H S Siqueira and J M A Rebello, Detection of the propagation of defects in pressurised pipes by means of the acoustic emission technique using artificial neural networks, Insight, 48 (1), pp 45-51, 2006.

[4]. R R Silva, D Mery and S D Soares, Evaluation of acoustic emission signal parameters for identifying the propagation of defects in pressurised tubes, Materials Evaluation, Vol 66, pp 493-500, 2008.

[5]. S D Soares, Correlações entre Emissão Acústica de Defeitos e Parâmetros de Mecânica da Fratura na Avaliação da Integridade de Equipamentos Pressurizados, doctoral thesis, Federal University of Rio de Janeiro, Rio de Janeiro, Brazil, 2008

[6]. B. P. Lathi, Modern Digital and Analog Communication Systems, Oxford University Press, 1988.

[7]. H. Sizun, Radio wave propagation for telecommunication applications, Springer, 2010.

[8]. M. H. Hassoum, Fundamentals of Artificial Neural Networks, The MIT Press. Cambridge, 1995.

[9]. S Haykin, Neural Networks - A Comprehensive Foundation, Macmillan College Publishing Inc, USA, 1994.

[10]. S. Samarasinghe, Neural Networks for Applied Sciences and Engineering - From Fundamentals to Complex Pattern Recognition, Auerbach Publications, USA, 2007.

[11]. I. N. da Silva, D. H. Spatti, R. A. Flauzino, Redes Neurais Artificiais para Engenharia e Ciências Aplicadas, Artliber Editora Ltda, Brasil, 2010 